\section{Cahiers de Narratologie}

Analyse et théorie narratives

8 | 1997

Création de l'espace et narration littéraire

\title{
L'espace dans Les regrets de Joachim Du Bellay
}

\section{Éliane Kotler}

\section{OpenEdition}

Journals

Édition électronique

URL : http://journals.openedition.org/narratologie/10761

DOI : 10.4000/narratologie.10761

ISSN : 1765-307X

Éditeur

LIRCES

Édition imprimée

Date de publication : 1 décembre 1997

Pagination : 193-208

ISBN : 291089746X

ISSN : 0993-8516

\section{Référence électronique}

Éliane Kotler, «L'espace dans Les regrets de Joachim Du Bellay », Cahiers de Narratologie [En ligne], 8| 1997, mis en ligne le 01 décembre 2020, consulté le 25 février 2021. URL : http://

journals.openedition.org/narratologie/10761; DOI : https://doi.org/10.4000/narratologie.10761

Ce document a été généré automatiquement le 25 février 2021.

Article L.111-1 du Code de la propriété intellectuelle. 


\title{
L'espace dans Les regrets de Joachim Du Bellay
}

\author{
Éliane Kotler
}

Parmi les poèmes que Du Bellay publie en 1558 à son retour de Rome, le recueil des Antiquitez, placé sous le signe de la peinture, nous invite à nous interroger sur l'espace ${ }^{1}$. Mais sommes-nous fondés à parler d'espace à propos d'un recueil beaucoup moins « descriptif » comme les Regrets ? Ce qui légitime le projet, c'est la dimension narrative sous-jacente du recueil, mais aussi le fait que la conception bellayenne de la poésie s'exprime en termes d'espace. En effet, l'espace des Regrets c'est d'abord l'espace de l'écriture, un espace poétique purement conceptuel et pourtant présent à tout esprit cultivé de l'époque de la Renaissance. L'espace des Regrets, c'est aussi le cadre d'un discours à la première personne, c'est-à-dire, en schématisant et en recomposant les divers espaces présents dans le recueil, l'espace du voyage qui a conduit notre poète aux rives du Tibre, celui de la ville de Rome où le poète a suivi son oncle le Cardinal Jean Du Bellay en mission et où il se sent en exil, et son contrepoint gaulois, lieu de nostalgie. C'est également un ailleurs du désir situé quelque part dans des régions éthérées, accessibles par l'esprit s'élevant au dessus des choses terrestres. Autant d'espaces différents traités de différentes façons. Dans leur évocation interviennent en effet à des degrés divers le poids de la culture et l'expérience vécue du poète qui prend tantôt la forme d'un regard jeté sur ce qui l'environne, tantôt celle d'une déambulation dans le cadre urbain de la Ville.

2 Il ne s'agira pas seulement pour nous de dire ce qui caractérise chacun de ces espaces mais de les réintégrer dans une géographie d'ensemble des Regrets, dont on mettra en lumière la cohérence et dont on essaiera de dégager la spécificité.

\section{L'espace conceptuel de l'écriture}

3 Depuis l'Antiquité, tout se passe comme si chaque entreprise de création poétique se devait de prendre place dans un univers conceptuel, hiérarchisé en termes d'espace, présent à l'esprit de toute personne lettrée ${ }^{2}$ : les poètes sont tenus de régler la 
« hauteur » de leur écriture sur celle du sujet choisi, le style élevé convenant aux sujets considérés comme nobles comme la théologie, les arts libéraux ou la politique, le style moyen à des sujets privés, aux arts mécaniques, à tout ce qui touche aux finances, le style bas à des sujets familiers, famille, enfants, fleurs et bergers ${ }^{3}$. Cette tripartition va de pair avec une axiologie: si les théoriciens conseillent d'éviter la boursouflure qui guette le style élevé, c'est ce dernier qui est l'objet de la plus grande considération, comme si les autres étaient en quelque façon subalternes. Cependant au XVI siècle, nombreux sont les poètes qui se targuent d'écrire dans un style bas qu'ils réhabilitent par là même. Quand Ronsard, dans les Amours de Marie, simple paysanne qu'on ne peut guère chanter qu'en style bas, prône la beauté de ce style ${ }^{4}$ et que Du Bellay dans ses Regrets revendique l'honneur d'écrire dans un style bas, ils ne font rien d'autre que renverser une axiologie bien établie mais que les poètes ressentent à présent comme étrangère à leur sensibilité.

Dans Les Regrets, la récurrence d'expressions qui visent à situer l'œuvre dans les régions basses de cet espace poétique conceptuel hiérarchisé est tout à fait frappante. Se comparant à Ronsard, le poète du style élevé, Du Bellay se déclare " agité d'une fureur plus basse » (S. 4). Et, toujours mu par la même volonté de se démarquer par rapport à son rival et néanmoins ami, il reprend la même idée dans le sonnet 60 :

... \& m'excusez de grâce,

Si pour le bal ayant la musique trop basse,

Je sonne un passe-pied, ou quelque branle gay.

$5 \quad$ Fait plus étonnant, l'éloge de grands personnages va se situer sur un terrain qui jusque là excluait de tels sujets : ce n'est pas dans la tonalité la plus élevée que le poète chante les louanges d'Avanson, ambassadeur du roi à Rome et dédicataire des Regrets, c'est au contraire, la « chorde la plus basse » qui est sollicitée pour cet office (S. 165).

Dans son refus d'une poésie "haute ", Du Bellay fait appel à deux images spatiales et culturelles également récusées, celle des hauteurs du Parnasse et celle de «l'onde au cheval », la source Hippocrène, elle-même située dans les régions hautes, à cause des références culturelles qui lui sont attachées:

Un plus sçavant que moy (Paschal) ira songer

Aveques l'Ascrean dessus la double cyme :

Et pour estre de ceulx dont on fait plus d'estime,

Dedans l'onde au cheval tout nud s'ira plonger.

7 Quant à la représentation de l'œuvre elle-même, ce sont des figures spatiales empruntées au registre de l'architecture qui en donnent une image dans les deux sonnets adressés à Clagny, architecte du Louvre :

Aux Muses je bastis d'un nouvel artifice

Un palais magnifique à quatre appartemens (S. 157)

De ce Royal palais, que bastiront mes doigts, (...)

Les ornemens seront de traicts \& d'arcs turquois. (S. 158)

8 L'œuvre poétique semble bien près de disparaître derrière sa représentation métaphorique, représentation bien peu compatible au demeurant avec le désir que le poète affiche par ailleurs de situer sa création dans les régions basses de l'univers poétique. Mais c'est bien là l'un des aspects de la poétique bellayenne, fondée pour une large part sur la contradiction ou les paradoxes ${ }^{5}$.

9 Mais, dans la perspective narratologique qui est la nôtre, il est intéressant de nous demander comment la personne du locuteur-poète s'inscrit dans cet univers de pure 
conceptualité. Et il est curieux de voir que le haut et le bas reparaissent à travers des images de déplacement. Là où Ronsard emprunte le "pénible sentier qui meine à la vertu », Du Bellay se sent contraint de rester dans « le chemin plus Batu» (S. 3) qu'il déconseillera pourtant au dauphin parce qu'il fait accéder «sans eschelle » au « séjour du vice » $(\mathrm{S} .172)^{6}$. Cet itinéraire poétique n'a rien d'un parcours nu où chacun suivrait sa propre voie, il est au contraire jalonné de modèles à suivre, de guides bons ou mauvais ou d'objectifs à atteindre qui, tout en structurant l'espace poétique conceptuel des Regrets, jettent les grandes lignes d'un art poétique sans que le poète ait l'air de théoriser. On note, en effet, la récurrence assez exceptionnelle de verbes qui expriment un mouvement ou impliquent une instance montrant le chemin. C'est ainsi qu'une prédestination d'origine ovidienne prend la forme d'un guide néfaste, comme le montre le sonnet 25 :

Vrayment d'un bon oiseau guidé je ne fus point, (...)

Mais tousjours le destin me tiroit au contraire

10 Cette idée que Fortune conduit le destin du poète est l'une de celles qui contribue à l'unité de l'œuvre puisqu'on la retrouvera exprimée dans l'un des derniers sonnets du recueil, le sonnet 189 :

Depuis ayant le cours de Fortune suivy

Ou le Tybre tortu de jaune se colore...

C'est aussi dans un univers fortement marqué par la mythologie qu'évolue le poète :

Je suivois d'Apollon la trace non commune, (...)

Et c'est pourquoy (Seigneur) ayant perdu la trace

Que suit vostre Ronsard par les champs de la Grace,

Je m'adresse où je voy le chemin plus batu :

Ne me bastant le cœur, la force, ny l'haleine

De suivre, comme luy, par sueur \& par peine

Ce penible sentier qui meine à la vertu. (S. 3)

Dans cet univers poétique structuré par différents modèles subis, choisis ou récusés qui se met progressivement en place au fur et à mesure que s'égrènent les sonnets, le modèle ronsardien est à la fois objet de fascination et de refus :

Ce pendant (mon Ronsard) pour tromper mes ennuys,

Et non pour m'enrichir, je suivray, si je puis,

Les plus humbles chansons de ta Muse lassee. (S. 22)

13 L'espace dont nous avons parlé jusqu'à présent est un espace purement conceptuel, dénué du moindre embryon de référence dans le monde réel tangible. Mais au sein des Regrets, l'image textuelle du poète s'inscrit aussi dans un autre espace faisant figure d'espace cadre dont l'origine essentiellement culturelle ne l'empêche pas de produire un certain effet de réel.

\section{L'ici et l'ailleurs : réalité et culture}

14 L'espace dans lequel évolue le poète se définit avant tout par opposition à un ailleurs regretté et idéalisé. L'opposition entre l'ici et l'ailleurs est nettement marquée, notamment par le balancement, souligné le plus souvent par la structure du sonnet, entre un arrière plan maintenu à distance (« cependant que » vous autres demeurés au giron de la mère patrie faites ceci), et un premier plan (moi, « icy » suis occupé à cela). Citons à titre d'exemple le sonnet 122 : 
Cependant qu'au Palais de procez tu devises, (...)

Nous devisons icy de quelques villes prises.

15 L'insistance sur le déictique " icy », qui peut, selon le contexte, faire référence à différents lieux, marque le lien entre un espace géographique donné et la personne du poète ; il suggère aussi, à lui seul ou avec un contrepoint plus temporel que spatial, un ailleurs ou un là-bas, lieu de tous ses désirs insatisfaits. En effet, même quand l'«icy » seul est nommé, comme dans le sonnet 127, la reprise du déictique, répété neuf fois dans le sonnet, nous apparaît comme un indice nous invitant à l'interpréter en fonction d'un contrepoint sous jacent :

Icy de mille fards la traison se desguise,

Icy mille forfaitz pullulent à foison,

Icy ne se punit l'homicide ou poison,

Et la richesse icy par usure est acquise :

Icy les grands maisons viennent de bastardise,

Icy ne se croid rien sans humaine raison,

Icy la volupté est toujours de saison,

Et d'autant plus y plaist, que moins elle est permise.

Pense le demourant. Si est-ce toutefois

Qu'on garde encor'icy quelque forme de loix,

Et n'en est point du tout la justice bannie :

Icy le grand seigneur n'achete l'action,

Et pour priver autruy de sa possession

N'arme son mauvais droit de force et tyrannie.

16 L'absence de cet ailleurs, vécue de façon douloureuse génère des images particulièrement belles où le paysage réel et le paysage intérieur se superposent comme dans le sonnet 8 :

Les costaux soleillez de pampre sont couvers,

Mais des Hyperborez les eternels hyvers

Ne portent que le froid, la neige, \& la bruine.

17 A l'ici est associée l'image mentale d'un paysage hivernal se superposant au paysage réel, méridional et ensoleillé ; et cette image mentale demeure dans le sonnet suivant où le poète écrit :

Entre les loups cruels j'erre parmy la plaine,

Je sens venir l'hyver, de qui la froide haleine

D'une tremblante horreur fait herisser ma peau.

Quant à l'ailleurs, le pays natal, il n'apparaît guère qu'à travers quelques figures culturelles plus que pittoresques ${ }^{7}$, en tout état de cause rassurantes, hautement symboliques et riches en connotations affectives, comme celle de la fumée s'élevant vers le ciel, suggérant l'image du foyer, lieu de rayonnement de chaleur, lieu de réunion, d'enracinement et de retour après l'éloignement ${ }^{8}$. Cette image n'apparaît pas seulement dans le célèbre sonnet Heureux qui comme Ulysse, comme le montre le sonnet 130 :

Et je pensois aussi ce que pensoit Ulysse

Qu'il n'estoit rien plus doulx que voir encor un jour

Fumer sa cheminee, et apres long sejour

Se retrouver au sein de sa mere nourrice.

Protection et fertilité caractérisent ce pays natal agreste, toujours associé au regret :

Je regrete les bois, et les champs blondissans,

Les vignes, les jardins et les prez verdissans,

Que mon fleuve traverse (S. 28) 
étroitement associé à la mer, une mer hostile et dangereuse. Alors qu'il est avéré que Du Bellay s'est rendu à Rome et en est revenu par voie terrestre, les Regrets évoquent un voyage maritime, assorti de tous les périls de la navigation et de la menace permanente du naufrage; témoin le sonnet 34 , où la mer semble soudain chavirer et de surface plane, devenir inquiétante verticalité :

Comme le marinier que le cruel orage

A long temps agité dessus la haute mer,

Aiant finablement à force de ramer

Garanty son vaisseau du danger du naufrage,

Regarde vers le port sans plus craindre la rage

Des vagues ny des vents, les ondes escumer :

Et quelqu'autre bien loing au danger d'abysmer

En vain tendre les mains vers le front du rivage :

Ainsi (mon cher Morel) sur le port arresté

Tu regardes la mer, \& vois en seureté

De mille tourbillons son onde renversee :

$\mathrm{Tu}$ la vois jusqu'au ciel s'eslever bien souvent,

Et vois ton Dubellay à la mercy du vent

Assis au gouvernail dans une nef persee.

Ce n'est pas seulement l'expression « mare altum », la mer profonde qui est à l'origine des représentations que nous avons ici. L'adjectif « haute » dénote ici véritablement la verticalité ascendante, puisque dans les tercets on voit «jusqu'au ciel s'eslever bien souvent » la « haute » mer dont il a été question au début du poème.

Mais ce motif culturel de la mer n'a pas seulement une valeur esthétique, la culture et la traditon rencontrent ici manifestement la psychologie du poète à l'esprit duquel toute situation mal vécue se présente spontanément sous la forme métaphorique de l'immensité marine. C'est ainsi que le locuteur-poète se décrit comme celui

...qui des flots marins longuement oultragé,

Tout moite et degoutant s'est sauvé du naufrage

Ce qui lui donne le droit d'adresser cette mise en garde à Ronsard :

Donques je t'advertis, que ceste mer Romaine

De dangereux escueils \& de bancs toute pleine

Cache mille perils, \& qu'icy bien souvent

Trompé du chant pippeur des monstres de Sicile

Pour Carybde eviter tu tomberas en Scylle,

Si tu ne sçais nager d'une voile à tout vent. (S. 26)

Les images de la mer peuvent donc faire référence à la tromperie et aux pièges du monde romain, mais plus généralement aux vicissitudes de la vie, aux difficultés quelles qu'elles soient. Il est alors curieux de constater que le poète se sent proche, voire solidaire de ceux qui éprouvent des revers de fortune comme le poète Baï :

Baif, qui, comme moy, prouves l'adversité,

Il n'est pas tousjours bon de combattre l'orage,

Il fault caler la voile, \& de peur du naufrage,

Ceder à la fureur de Neptune irrité. (S. 56)

ou comme le cardinal Du Bellay, victime d'accusations injustes : 
Je me console donc, \& en pareille mer,

Voiant mon cher Seigneur au danger d'abysmer,

Il me plaist de courir une même fortune. (S. 49)

Cette mer de tous les dangers c'est la mare nostrum des poètes grecs et latins ; une mer qui s'oppose à l'océan qui, lui, semble ne faire qu'un avec la terre qu'il prolonge et avec laquelle il se confondrait presque dans une belle image exprimant le désir du poète de revoir « de l'ocean les campagnes humides » (S. $128^{9}$.

Dans un tel contexte, on ne sera pas surpris que le voyage ne se conçoive que comme déplacement « de port en port » (S. 30). De fait, Du Bellay n'arrive pas à Rome, mais au « rivage Romain » (S. 131) ; quant au lieu de séjour, c'est un lieu frontière, «bord » ou " rivage ", le plus souvent connoté négativement, à travers des images de rejet et de stérilité véhiculées par des adjectifs comme «estranger $»^{10}$, le plus employé, " avare $»^{11}$ ou " infertile $»^{12}$. Avec une certaine redondance, le «bord» et le « rivage» peuvent d'ailleurs être associés dans un même vers, comme dans le sonnet 16 où leur association suggère véritablement un point extrême appelant des images de mort :

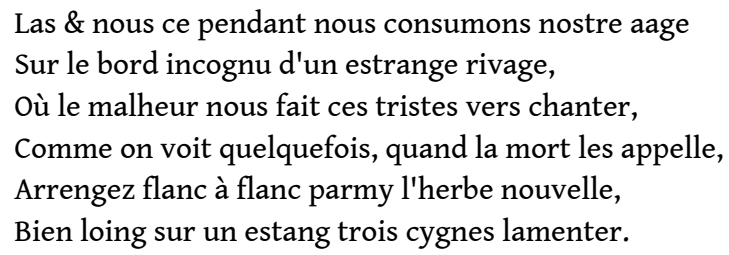

Cette association que l'on pourrait croire fugitive apparaît également dans le sonnet suivant, le sonnet 17 , où le « bord » se situe alors très explicitement du côté de la mort.

Il est clair pour tous et encore davantage pour les contemporains de Du Bellay que les origines de ces images sont culturelles. On trouve des échos de l'Odyssée et de l'Enéide dans l'espace maritime des Regrets ${ }^{13}$ et des éléments de la navigation des héros de ces deux épopées émergent du recueil de Du Bellay comme des signes reconnaissables d'un espace géographique englobant, commun aux grands modèles littéraires de l'Antiquité gréco-latine. Ce fonds culturel fait figure de superstructure définissant un espace poétique plus large que celui d'une œuvre ancrée dans un siècle déterminé, laquelle se trouve par ce biais inscrite dans une tradition et une lignée prestigieuses. L'autre source majeure, sous-jacente dans tout le recueil, c'est Ovide qu'une communauté de pensée unit à Du Bellay, et dont l'exil véritable aux bords du Pont Euxin se trouve transposé au rivage romain.

Cependant le poids de la culture ne suffit pas à expliquer la présence quasi obsessionnelle du motif du bord ou du rivage. Pour résoudre ce problème, sans doute nous faut-il considérer que cet espace géographique particulier est à l'image de l'espace intérieur du poète, espace lumineusement éclairé par cette observation de Bakhtine, un peu abusivement généralisée à l'espèce humaine dans son ensemble : «L'homme ne possède pas de territoire intérieur souverain, il est entièrement et toujours sur une frontière $\aleph^{14}$. Ces images de zones limites, de lisières figurent en effet la fragilité, la précarité, réelle ou vécue comme telle, de la situation dans laquelle se trouve Du Bellay. Ce qui légitime cette interprétation, c'est que, s'ils sont le plus souvent employés pour évoquer l'exil, les termes appartenant au champ lexical du bord, de la rive, de la frontière ne sont pas réservés à cet emploi, mais peuvent aussi désigner la patrie originelle; c'est le cas dans le sonnet 27, où deux images étrangement voisines et antithétiques se succèdent, celle de la "rive paternelle », connotée positivement, et 
celle du "bord estranger » sur lequel le poète vit «languissant». Plus loin, c'est un retour au « rivage gaulois » que le poète envisagera par l'esprit (S. 128).

Cependant il est évident que ces deux espaces frontières ne sont pas situés de la même façon par rapport à la personne du locuteur-poète. Quand la rive paternelle est affectée d'une forme non marquée du déterminant ( J'arresteray ma nef au rivage Gaulois », S. 128) ou d'un adjectif possessif qui marque la relation affective (" ma rive paternelle», S. 27), le rivage romain, lui, est le plus souvent déterminé par un adjectif démonstratif, déictique spatial indiquant à la fois la proximité et le rejet affectif («ce bord estranger ", S. 27, " ces flots tortueux », " ces flots estrangers ", S. 128). S'il arrive que la valeur de déictique soit prépondérante, comme lorsque Du Bellay oppose «ceste Rome cy » à " ceste Rome la » qui avait eu l'heur de séduire Robertet (S. 83), il est plus fréquent que l'axiologie se mêle à la désignation, comme dans les sonnets 17 « ceste plage ", 28 « ce bord », 37 « ce bord estranger ", et bien d'autres encore.

Cet espace culturel et presque archétypal côtoie pourtant un autre espace dont on peut dire qu'il est plus réel dans la mesure où il admet un référent authentifiable qui ne soit pas purement livresque. Cependant l'évocation de cet espace ne prend pratiquement jamais l'allure d'une description; l'espace qui se dessine sous ses yeux du lecteur est soit médiatisé par le regard du locuteur-poète, soit parcouru dans toutes ses dimensions par la déambulation, soit enfin rejeté par l'aspiration à la fuite.

\section{Le poète et l'espace : regard, parcours et fuite}

Ces trois attitudes du locuteur-poète définissent trois modes différents d'appréhension de l'espace dont le premier est dominé par le regard. La récurrence du verbe "voir " dans les Regrets est en effet tout à fait remarquable; sauf erreur, nous avons comptabilisé 104 emplois où le verbe "voir ", rapporté au sujet de l'énonciation ou l'englobant, se tourne vers le monde extérieur ${ }^{15}$. On notera par ailleurs que ce verbe n'admet aucun substitut.

L'espace romain est avant tout perçu comme un spectacle dont le poète nous donne une vision satirique. La Rome du XVI ${ }^{\mathrm{e}}$ siècle est bien là avec ses prostituées (S. 131), sa cour pontificale (S. 118), l'ascension puis la chute ou la mort des papes (S. 113), l'agitation d'une nation prête à entrer en guerre (S. 116), ses fêtes « romanesques ", en particulier celles données à l'occasion du Carnaval (S. 120 et 121). A travers le regard du poète le lecteur découvre aussi, au détour d'une réflexion eschatologique, ce qui caractérise de nombreuses villes dotées d'un passé ancien, à savoir la superposition des constructions sur un même site :

Et qui sçait si les Cieulx referont point leur tour,

Puis que tant de Seigneurs nous voyons chacun jour

Bastir sur la Rotonde, \& sur le Collisee ? (S. 107)

Si la médiation du sujet de l'énonciation est largement dominante, il arrive pourtant qu'exceptionnellement la relation de la chose vue ne soit pas directe et que le poète délègue son pouvoir de parole, comme dans le sonnet 119:

Brusquet à son retour vous racontera (Sire)

De ces rouges prelatz la pompeuse apparence,

Leurs mules, leurs habitz, leur longue reverence,

Qui se peult beaucoup mieulx representer que dire. 
C'est essentiellement Rome qui est l'objet du coup d'œil faussement surpris et authentiquement indigné du poète, mais d'autres villes comme Venise n'échappent pas à son regard satirique :

Il fait bon voir (Magny) ces Coions magnifiques, Leur superbe Arcenal, leurs vaisseaux, leur abbord, Leur sainct Marc, leur palais, leur Realte, leur port, Leurs changes, leurs profitz, leur banque, \& leurs trafiques :

Il fait bon voir le bec de leurs chapprons antiques, Leurs robbes à grand'manche...

Le regard ne prétend jamais à l'exhaustivité mais à travers quelques images métonymiques faisant figure de clichés caractéristiques, le lecteur parvient à se représenter l'espace urbain où évolue le poète.

L'autre attitude du poète dans sa relation avec l'espace romain consiste à le parcourir. "Quand je vays par la rue ", écrit-il dans le sonnet 99. Mais c'est le sonnet 80 qui nous donne l'image la plus complète de cette déambulation romaine :

Si je monte au Palais, je n'y trouve qu'orgueil,

Que vice desguisé, qu'une cerimonie,

Qu'un bruit de tabourins, qu'une estrange armonie,

Et de rouges habits un superbe appareil :

Si je descens en banque, un amas \& recueil

De nouvelles je treuve, une usure infinie,

De riches Florentins une troppe banie,

Et de pauvres Sienois un lamentable dueil :

Si je vais plus avant, quelque part ou j'arrive,

Je treuve de Venus la grand'bande lascive

Dressant de tous costez mil appas amoureux :

Si je passe plus oultre, \& de la Rome neufve

Entre en la vieille Rome, adonques je ne treuve

Que de vieux monuments un grand monceau pierreux.

offert au regard du poète ou parcouru par lui, l'espace cadre des Regrets c'est avant tout Rome et son contrepoint gaulois, mais on observera aussi la présence de quelques villes comme Urbino, Ferrare, Venise, du côté italien ${ }^{16}$, Lyon ou Paris, du côté français ${ }^{17}$, ainsi que du pays helvète ${ }^{18}$; villes et paysages font l'objet d'évocations rapides mais parlantes; nul doute que Du Bellay sait l'art de relever dans tous les cas le détail typique qu'il se plaît à tourner en dérision.

L'espace « réel » des Regrets, l'espace romain en particulier, apparaît en définitive comme un véritable espace scénique dans lequel le poète serait tantôt acteur, déambulant par les rues de Rome, tantôt spectateur voyant se dérouler sous ses yeux des scènes qui stimulent sa plume satirique.

d'un œil critique en appelle un autre, virtuel, qui se présente au lecteur comme l'espace du désir. Les appels à la fuite sont nombreux; citons presque au hasard le sonnet 50 :

Sortons (Dilliers) sortons...

Et fuions desormais ce tumulte civil,

Sus donc (Gordes) sus donc, à la voile, à la rame,

Fuions, gaignons le hault, je voy la belle Dame

Qui d'un heureux signal nous appelle à son port.

le 116 : 
Fuions (Dilliers) fuions ceste cruelle terre,

Fuions ce bord avare, \& ce peuple inhumain l'espace poétique purement conceptuel tel que l'ont suggéré les premiers sonnets où le poète donnait un cadre à son projet poétique. Tout se passe comme si le projet poétique et la démarche même du poète s'intégraient dans deux espaces différents, mais de structure isomorphe.

51 Au terme de cette étude, on remarquera tout d'abord la place prépondérante accordée aux représentations verticales : qu'il s'agisse du métadiscours spatialisé de l'écriture ou de l'univers du désir, tous deux figurent un espace marqué par la verticalité, où même ce qui est horizontal comme la mer tend à devenir vertical. On notera aussi l'épure, l'exceptionnelle stylisation de la cartographie d'ensemble de l'univers poétique des Regrets : peu de lignes, des images de zones frontière peu variées dans leur expression, peu d'attitudes différentes du locuteur-poète au sein de cet univers ${ }^{19}$. Enfin, un dernier constat s'impose, c'est que des éléments issus d'un fonds culturel commun à tous les poètes de la Renaissance, recomposés, dessinent un espace unique, appartenant en propre à Du Bellay. Il était entendu dès le début de notre étude que l'espace des Regrets ne pouvait être une représentation du monde réel, mais Du Bellay a su se réapproprier à la fois l'apport culturel tiré de l'Antiquité et les spectacles qui ont pu s'offrir à lui en 
les rapportant les uns et les autres à sa propre subjectivité, d'où l'unité du recueil et sa spécificité. Comme nous l'avons mentionné plusieurs fois au fil de cette étude, la culture antique de Du Bellay est à l'origine de la majeure partie des représentations spatiales des Regrets; mais ces représentations sont choisies en fonction d'un dessein d'ensemble, tout comme la place que le poète s'assigne lui-même au sein de son propre univers poétique, mais aussi d'un univers beaucoup plus vaste qui l'inclut. L'espace le plus rattaché au réel, l'espace romain, médiatisé par le regard ou la pérégrination se referme également de quelque façon sur le poète lui-même plutôt qu'il ne s'ouvre vers l'extérieur. Il ressort donc de tout cela que l'espace nous ramène sans cesse à la personne du poète, dont il est comme un miroir de son univers intérieur façonné par une culture personnelle puisée aux sources de la tradition. Sans doute cette démarche n'est-elle pas originale, mais son résultat, la représentation d'un espace typiquement bellayen, est quant à lui incontestablement unique.

\section{NOTES}

1. C'est ce qu'a fait Françoise Giordani dans son article intitulé «Utilisation et description symboliques de l'espace dans les Antiquitez de Rome de Joachim Du Bellay », dans Du Bellay et ses sonnets romains, Études sur les Regrets et les Antiquitez de Rome réunies par Yvonne Bellanger, Paris, Champion, Unichamp, 1994, p. 19-46.

2. Voir en particulier la Rhétorique à Hérennius, IV, 11, trad. G. Achard, coll. Budé ou Cicéron, l'Orateur, 69 ss.

3. Pour l'époque de la Renaissance, voir Pierre Fabri, le Grand et vray art de pleine rhétorique (1521), Slatkine 1969, texte établi à partir de l'édition de Rouen de 1889-1890.

4. ... les amours ne se souspirent pas

D'un vers hautement grave, ains d'un beau stille bas,

Elegie à son livre, v. 173-174, éd. Laumonnier, VII, p. 324.

5. Yvonne Bellenger a fort justement observé le phénomène dans son article « Du Bellay le versatile, Contradictions d'un grand poète ", dans Perspectives, Revue de l'Université Hébraïque de Jérusalem, Le Langage et le texte, Hommage à Alexandre Lorian, n² 2, 1995, p. 158-169.

6. L'univers mythique de la Renaissance éclaire ces images: la vertu serait logée au sommet d'une montagne.

7. Comme l'observe Philippe Hamon, au XVI ${ }^{\mathrm{e}}$ siècle, «Décrire, ce n'est jamais décrire un réel, c'est faire la preuve de son savoir-faire rhétorique, la preuve de sa connaissance des modèles livresques, où les officinae, les florilèges, les polyantheae ont déjà découpé, à des fins pédagogiques, les premières listes de morceaux choisis descriptifs pris aux meilleurs auteurs de l'antiquité. », Du Descriptif, Paris, Hachette Supérieur, 1993, p. 13.

8. La symbolique du foyer, déjà présente chez Homère, est reprise par Ovide (Les Pontiques, I, III, v. 33-34), puis par Marot. Voir à ce sujet, Henri Chamard, Histoire de la Pléiade, Paris Didier, 1961 (1 ${ }^{\mathrm{e}}$ éd. 1939), t. II, p. 245 , note 1.

9. Je remercie Marie-Hélène Cotoni d'avoir attiré mon attention sur l'existence de deux éléments marins antithétiques dans les Regrets.

10. Sonnets 10, 27, 37.

11. Sonnet 116. 
12. Sonnet 46.

13. Pour le détail de ces sources, on consultera avec profit les notes de l'édition Droz dont le texte, établi par J. Jolliffe, est introduit et commenté par M. A. Screech, Genève, Droz, 1966.

14. Cité par Todorov, Le principe dialogique, Paris, Seuil, 1981, p. 148.

15. Nous n'avons donc pas comptabilisé les formes pronominales qui n'ouvrent pas sur l'espace, mais se referment sur le sujet, de même que celles dont l'objet n'est autre que le poète lui-même. En revanche nous avons admis toutes les formes dont l'agent est suffisamment englobant ou suffisamment vague pour ne pas exclure le locuteur-poète.

Pour établir ce décompte, nous avons utilisé l'index figurant dans l'édition électronique des Regrets, établie par Christine de Buzon et Marie-Luce Demonet, EQUIL XVI, Université Biaise Pascal, Champion, 1994.

Cette récurrence du verbe "voir ", tout à fait frappante, n'est pas propre au recueil des Regrets, elle s'observe également dans les Antiquitez (voir à ce sujet Françoise Giordani, art. cit. dans la note 1, p. 22).

16. Sonnets 132 et 133.

17. Sonnets 137 et 138.

18. Sonnets 135 et 136 .

19. En marge de la question de l'espace on se demandera si la modernité du recueil n'est pas précisément là, dans cette pureté des lignes de force du recueil, assez exceptionnelle au milieu du $\mathrm{XVI}^{\mathrm{e}}$ siècle, modernité qui fait que cette poésie parle encore au lecteur d'aujourd'hui sans que ce dernier ait besoin de gloses explicatives.

\section{AUTEUR}

\section{ÉLIANE KOTLER}

Université de Nice-Sophia Antipolis 